\title{
ALTERAÇÕES PODAIS EM IDOSOS: REVISÃO INTEGRATIVA
}

\author{
Joziane Santos da Silva \\ Fátima Helena Espírito Santo ${ }^{2}$ \\ Carla Lube de Pinho Chibante ${ }^{3}$
}

resumo

Compreendendo a magnitude do cuidado integral à saúde da população idosa e considerando o quanto as alterações nos pés podem interferir nas atividades de vida diária, e consequentemente na qualidade de vida desses idosos, o presente estudo teve como objetivo: identificar na literatura evidências científicas acerca das alterações mais frequentes nos pés dos idosos. Levantou-se como questão da pesquisa: Como a literatura tem abordado o tema alterações nos pés dos idosos? Trata-se de uma Revisão Integrativa, e para tal foi realizada busca de artigos publicados e indexados na Biblioteca Virtual em Saúde utilizando a combinação dos seguintes Descritores em Ciên-

1 Enfermeira. Discente do Mestrado Profissional em Enfermagem Assistencial da Escola de Enfermagem Aurora de Afonso Costa (EEAAC/UFF). E-mail: jozysilva78@gmail.com.

2 Enfermeira. Professora Doutora do Departamento de Enfermagem Médico-Cirúrgica da Escola de Enfermagem Aurora de Afonso Costa (EEAAC/UFF). E-mail: fatahelen@hotmail.com.

3 Enfermeira. Doutora em Ciências do Cuidado em Saúde. Professora convidada do Curso de Especialização em Enfermagem Gerontológica da Escola de Enfermagem Aurora de Afonso Costa (EEAAC/UFF). E-mail: carla-chibante@hotmail.com. 
cias da Saúde em língua portuguesa: "Pé" e "Idoso" acompanhados do operador booleano "and". O resultado final deste levantamento é constituído por dez artigos publicados entre os anos de 2008 e 2017 em duas bases de dados: Literatura Latino-Americana e do Caribe em Ciências da Saúde (LILACS) e Medical Literature Analysis and Retrieval System on-line (MEDLINE). Os artigos foram divididos em duas categorias: Alterações nos pés relacionadas à incapacidade no idoso; Hospitalizações em decorrência de alteração podal: o pé diabético na população idosa. As alterações nos pés dos idosos são frequentes e podem ocorrer tanto como resultado de maus tratos aos pés, como uso de calçados inadequados e traumas, como em consequência de doenças como diabetes mellitus. As alterações podais mais frequentes foram hálux valgo, deformidades nos pequenos artelhos, calosidades, ressecamento, fissuras, unhas quebradiças, encravadas e compridas. Estas alterações podem comprometer a mobilidade e até o favorecer as quedas, colocando em risco a independência dos idosos.

palavras-chave

Pé. Idoso. Enfermagem Geriátrica.

\section{Introdução}

O número de idosos no país segue crescendo de forma acelerada. Dados do Instituto Brasileiro de Geografia e Estatística (IBGE) apontam que o número de idosos passou de 19,6 milhões em 2010, e deve tingir 41,5 milhões em 2030 e 73,5 milhões em 2060 (ERVATTI; BORGES; JARDIM, 2015).

O aumento da população de idosos evidencia maiores desafios para órgãos governamentais e para a sociedade em geral, especialmente para países em desenvolvimento. Na área da saúde o aumento desse seguimento da população exige dos profissionais e das instituições um melhor preparo no atendimento a essa população.

A trajetória profissional na área da enfermagem despertou-nos o interesse pela assistência aos idosos que são portadores de alterações nos pés, especialmente aqueles que desenvolveram alterações que possivelmente estejam relacionadas às patologias de base, como hipertensão, diabetes, neoplasias, entre outras. 
O pé é uma unidade funcional importante no controle da postura, manutenção do equilíbrio e na execução dos movimentos. Essas funções dependem da integridade anatômica e funcional de suas estruturas que, com frequência, são alteradas com o envelhecimento (AIKAWA et al., 2009).

Com o envelhecimento ocorrem alterações musculoesqueléticas nos membros inferiores que são consideradas a maior causa de incapacidade em idosos. Nos pés essas alterações podem ser decorrentes de doença sistêmica, transtornos da marcha, traumatismos e maus tratos que comprometem a integridade das unhas, da pele, dos nervos e da estrutura óssea (BARBOSA et al., 2015).

Em nossa atuação profissional enquanto enfermeira e podóloga, foi possível adquirir conhecimento ao vivenciar em várias situações o empenho para o desenvolvimento de ações que vão ao encontro das necessidades da população idosa. Porém, foi possível também perceber brechas que impedem que a assistência seja realizada de forma integral aos idosos, como a pouca atenção dada às alterações nos pés dos idosos que contribui para a falta de cuidado integral ao idoso. Alguns autores citam o foco exclusivo na doença como um dos problemas da maioria dos modelos assistenciais contemporâneos. Porém, o foco deve estar em ações de educação, promoção da saúde, prevenção de doenças evitáveis, postergação de moléstias, cuidado precoce e reabilitação (VERAS; OLIVEIRA, 2016).

Neste contexto ressalta-se a falta de cuidados específicos com os pés, que a despeito de desempenharem importante função na sustentação, no equilíbrio e na locomoção do corpo humano, muitas vezes são esquecidos.

Sabe-se que as alterações nos pés podem influenciar a vida dos idosos, contribuindo para a ocorrência de dores, maior risco de quedas e para o desenvolvimento do temido processo de incapacidade e dependência no idoso (MARIN; MACIEL, 2014). Portanto, é necessária a organização das instituições e capacitação dos profissionais de saúde para que possam atender às demandas apresentadas por esses idosos.

As alterações nos pés dos idosos podem ser decorrentes maus tratos, transtornos da marcha, de doenças sistêmicas e traumatismos nos pés que afetam a integridade das unhas, da pele, dos nervos, dos vasos e das estruturas ósseas, como o uso de sapatos de salto alto e bico fino. Estudos apontam que halux valgo, deformidades nos pequenos artelhos, espessamento e calosidades são as alterações mais comuns nos pés de idosos. Estas alterações causam dor, afetam a mobilidade e o equilíbrio e contribuem para um maior risco de quedas. Além disso, os pacientes que apresentam problemas nos pés têm maior probabilidade de relatar dores nas costas, quadris, joelhos e mãos, que 
também estão associados à diminuição do equilíbrio e maior risco de quedas (BARBOSA et al., 2015; MARIN; MACIEL, 2014).

Compreendendo a magnitude do cuidado integral à saúde da população idosa e considerando o quanto as alterações nos pés podem interferir nas atividades de vida diária (AVDs) e consequentemente na qualidade de vida desses idosos, o presente estudo se propõe a avaliar o conhecimento científico produzido acerca das alterações nos pés dos idosos.

Assim, levantou-se como questão da pesquisa: Como a literatura tem abordado as alterações nos pés dos idosos?

Este estudo teve como objetivo identificar na literatura evidências científicas acerca das alterações mais frequentes nos pés dos idosos.

\section{Método}

Trata-se de revisão integrativa cujo objetivo é agrupar e resumir os achados de pesquisas relacionadas a um determinado tema de forma sistemática e ordenada, levando o leitor à compreensão do tema estudado (MENDES; SILVEIRA; GALVÃO, 2008). E para a realização desta revisão foram seguidas as etapas de acordo com as orientações deixadas pelos autores.

Foi realizada busca de artigos publicados e indexados na Biblioteca Virtual em Saúde (BVS) utilizando a combinação dos seguintes Descritores em Ciências da Saúde (DeCS) em português: "Pé" e "Idoso" acompanhados do operador booleano "and".

Os critérios de inclusão definidos para a seleção das produções científicas foram: textos publicados na íntegra, gratuitos, que retratassem a temática referente às alterações nos pés, em língua portuguesa e/ou espanhola, publicados e indexados nas bases de dados. Estabeleceu-se como critério de exclusão, artigos que se repetiram nas bases de dados.

A busca foi realizada no mês de dezembro de 2019 e foram encontradas 24.960 produções. Aplicando o filtro idioma restaram 1.150 produções (648 em Espanhol e 507 em Português) e destes, 586 estavam disponíveis na íntegra. Ao aplicar o filtro artigo restaram 125 produções (58 em português e 67 em espanhol). Quanto às bases de dados 62 artigos estavam incluídos na LILACS, 41 na MEDLINE, 13 na IBECS, 4 na BDENF , 1 na CUMED, 1 na Odontologia e 1 na Hanseníase, sendo que 2 artigos se repetiram nas bases de dados. No total foram 123 artigos disponíveis. Após a leitura dos títulos dos artigos, foram excluídos 84 por não abordarem o tema proposto para esta RI. Procedeu-se a leitura dos resumos dos 39 artigos restantes, sendo excluídos 26, pois não 
abordavam a temática em questão. Então restaram 13 artigos para a leitura e destes, 3 foram excluídos por abordarem adultos jovens e terem em suas amostras um número de idosos pouco expressivo.

\section{Resultados}

Apesar de não ter sido designado um recorte temporal para a realização da busca das produções científicas, a seleção dos artigos que atendiam aos critérios previamente estabelecidos trouxe produções recentes, todas publicadas entre 2008 e 2017, sendo 1(10\%) do ano de 2017, 2 (20\%) do ano de 2015, 1(10\%) de 2014, 2 (20\%) de 2012, 1(10\%) de 2011, 1 (10\%) de 2009 e 2 (20\%) de 2008.

Dos dez estudos selecionados, 9 (90\%) foram desenvolvidos no Brasil e 1 (10\%) foi realizado em uma Instituição de Longa Permanência para Idosos (ILPI) na Espanha. Dos 9 estudos desenvolvidos no Brasil 7 (70\%) foram realizados em ambulatórios, 1 (10\%) em uma Unidade de Saúde da Família e 1 (10\%) em uma unidade de internação hospitalar. Observou-se maior número de estudos com abordagem quantitativa, que aparece em 9 estudos (90\%) 8 no Brasil e 1 na Espanha. Apenas 1 estudo (10\%) teve abordagem qualitativa. Houve predominância também de pesquisas descritivas com 7 estudos (70\%). Estudos do tipo Exploratório, transversal e descritivo/exploratório/analítico tiveram 1 artigo cada . Quanto às bases de indexação, 1 artigo foi publicado na MEDLINE e 9 na LILACS. Por fim, todos os estudos tiveram nível de evidência científica IV segundo a Classificação de Oxford Centre for Evidence-Based Medicine.

A partir da análise dos artigos selecionados, apresentam-se no Quadro 1 os resultados quanto ao título, autor, ano e país de publicação, base de dados, delineamento do estudo, nível de evidência, instrumentos utilizados, principais resultados e conclusão. 


\begin{tabular}{|c|c|c|c|}
\hline 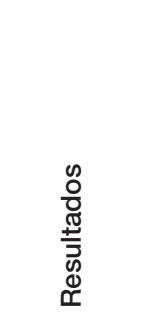 & 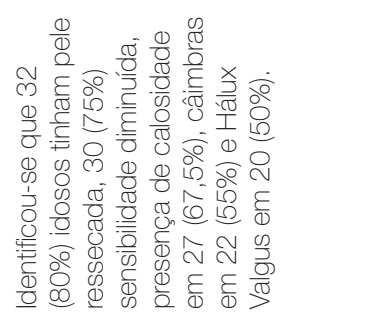 & 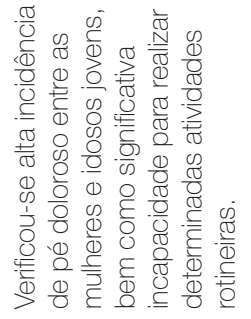 & 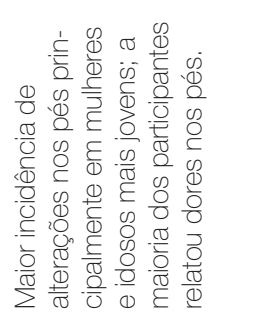 \\
\hline 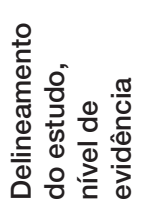 & 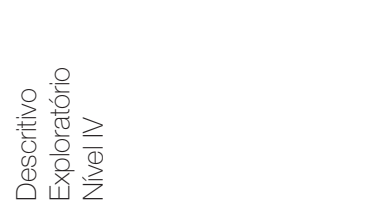 & 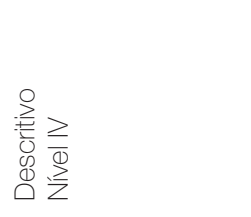 & 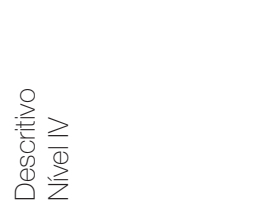 \\
\hline 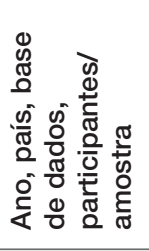 & 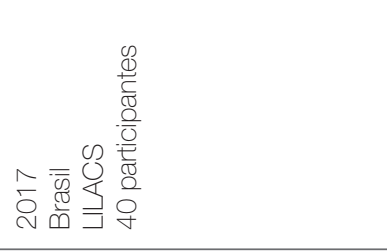 & 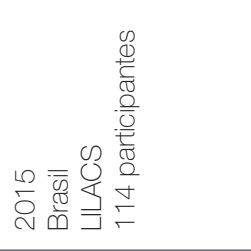 & 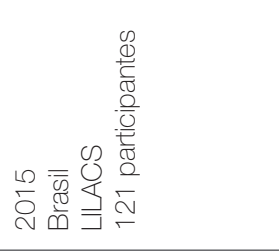 \\
\hline $\begin{array}{l}\stackrel{0}{2} \\
\frac{0}{0} \\
0\end{array}$ & 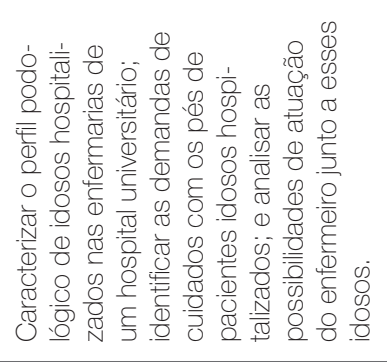 & 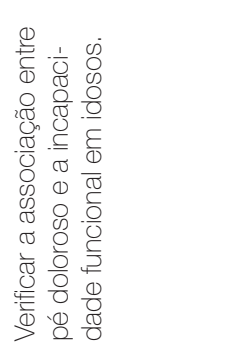 & 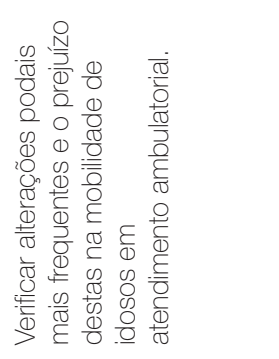 \\
\hline $\begin{array}{l}\text { @ } \\
\frac{0}{0} \\
\frac{3}{2}\end{array}$ & 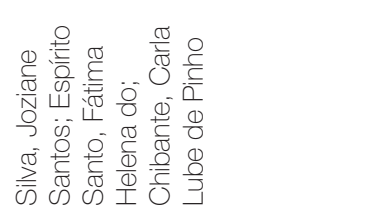 & 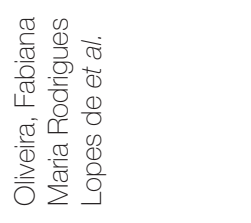 & 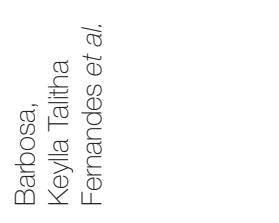 \\
\hline $\begin{array}{l}\frac{0}{2} \\
\stackrel{ \pm}{=}\end{array}$ & 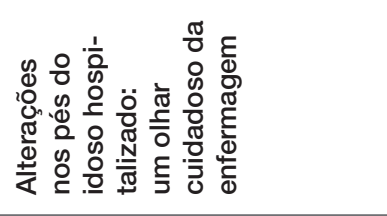 & 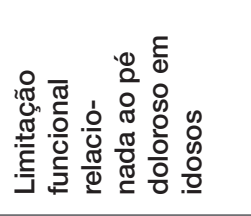 &  \\
\hline$\stackrel{\circ}{z}$ & 5 & ชิ & 8 \\
\hline
\end{tabular}




\begin{tabular}{|c|c|c|}
\hline 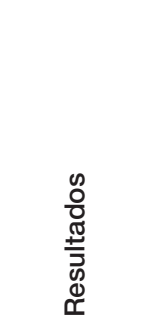 & 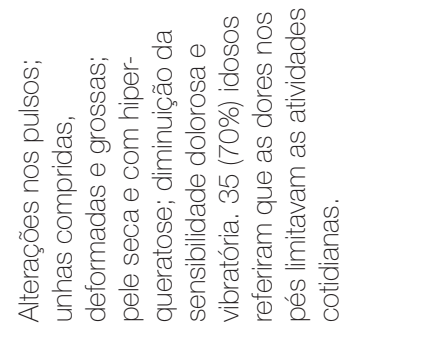 & 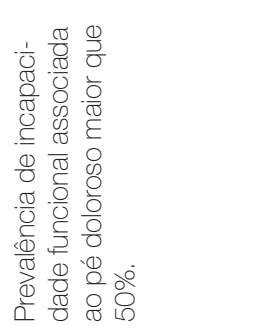 \\
\hline 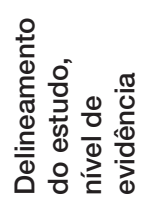 & 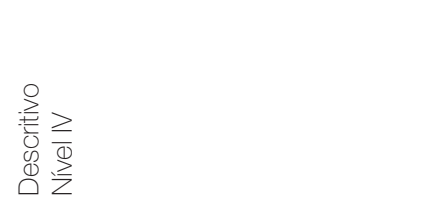 & 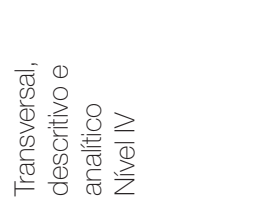 \\
\hline 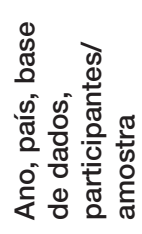 & 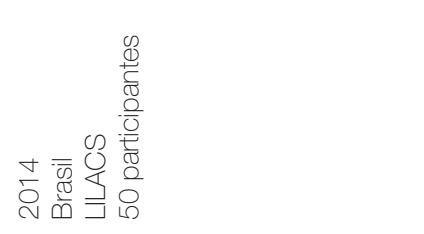 & 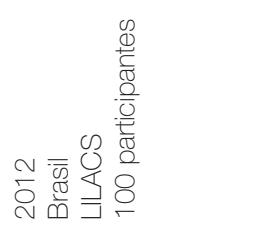 \\
\hline $\begin{array}{l}\frac{0}{\stackrel{0}{0}} \\
\frac{0}{0} \\
\frac{0}{0}\end{array}$ & 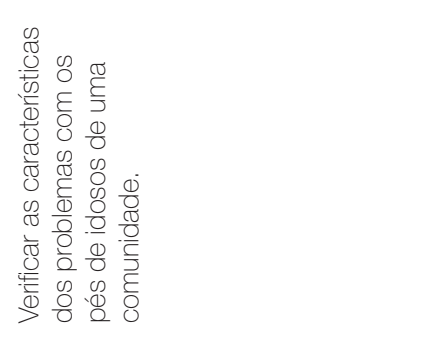 & 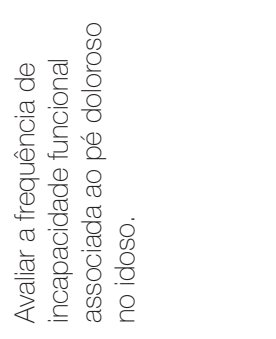 \\
\hline 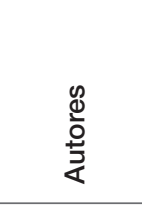 & 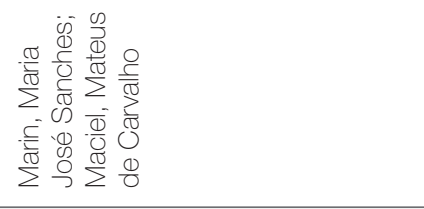 & 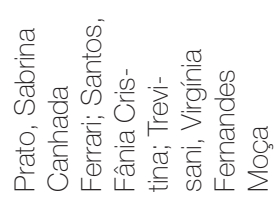 \\
\hline$\stackrel{\text { 을 }}{\frac{1}{2}}$ & 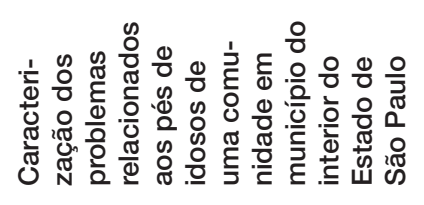 & 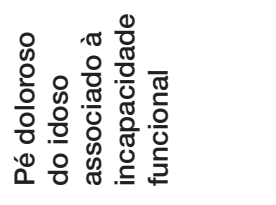 \\
\hline$\stackrel{\circ}{z}$ & t & ஜூ \\
\hline
\end{tabular}




\begin{tabular}{|c|c|c|}
\hline 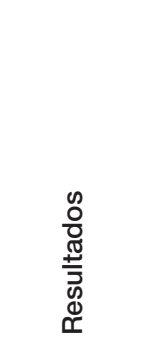 & 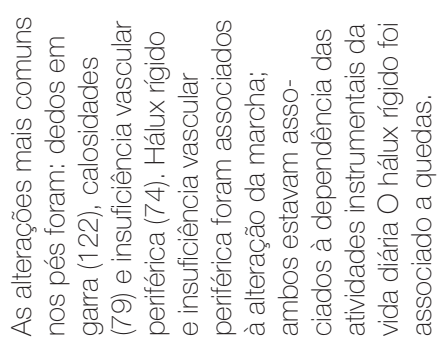 & 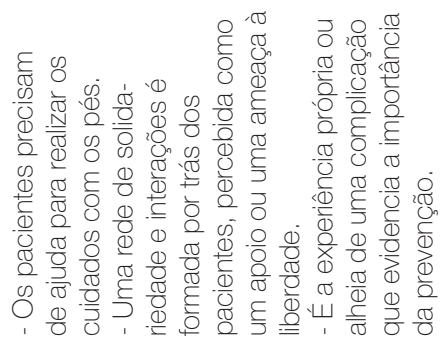 \\
\hline 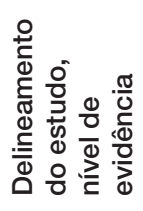 & 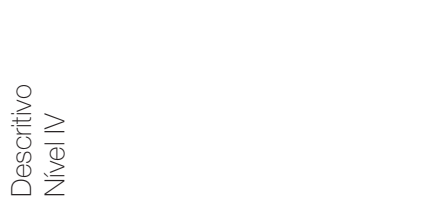 & 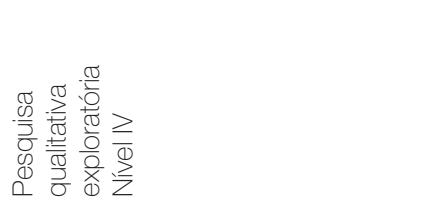 \\
\hline 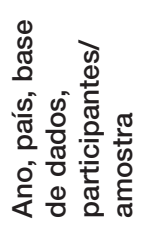 & 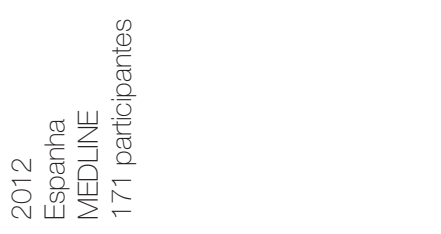 & 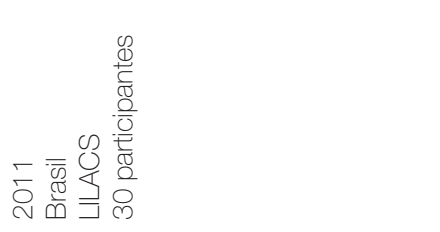 \\
\hline $\begin{array}{l}\stackrel{0}{3} \\
\frac{0}{0} \\
\frac{0}{0}\end{array}$ & 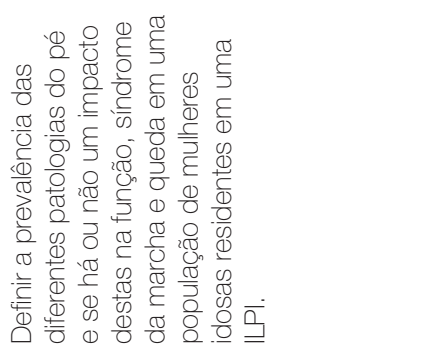 & 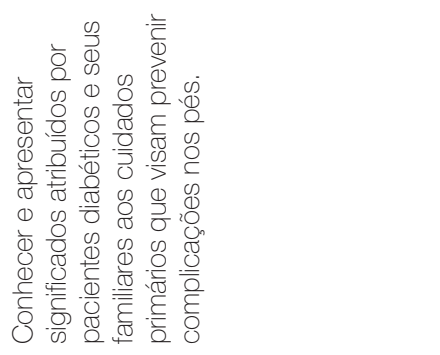 \\
\hline $\begin{array}{l}\text { d } \\
\frac{0}{0} \\
\frac{1}{3}\end{array}$ & 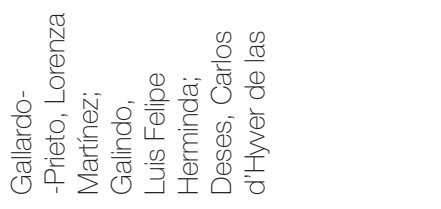 & 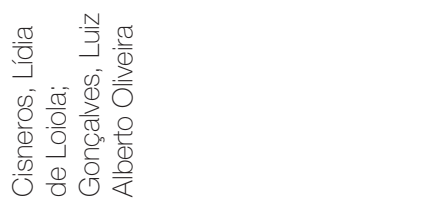 \\
\hline$\underset{ }{\frac{O}{3}}$ & 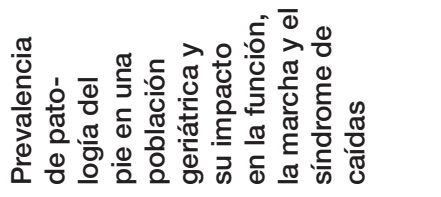 & 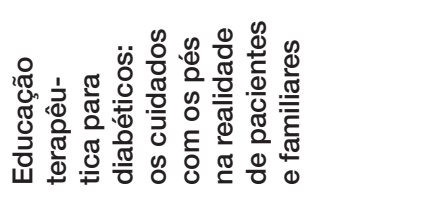 \\
\hline$\stackrel{\circ}{Z}$ & $\mathscr{\circ}$ & ⿵人 \\
\hline
\end{tabular}




\begin{tabular}{|c|c|c|}
\hline 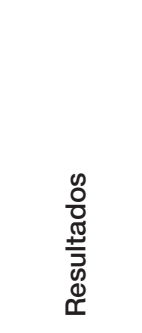 & 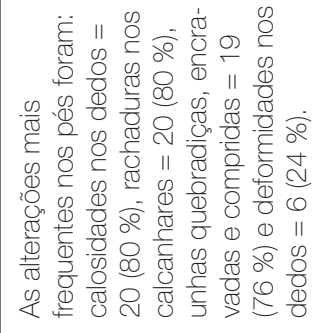 & 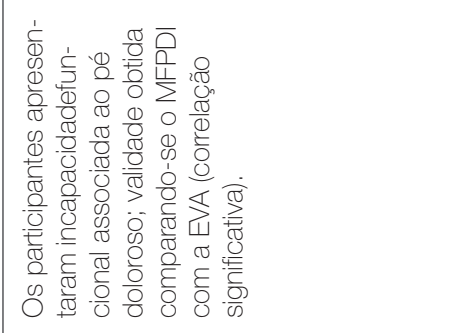 \\
\hline 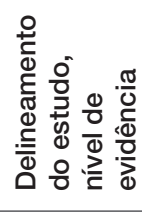 & 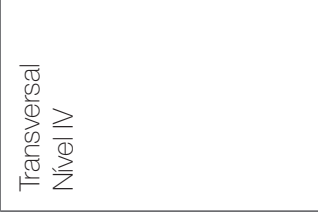 & 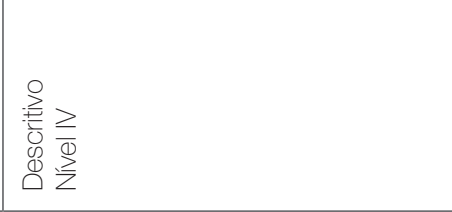 \\
\hline 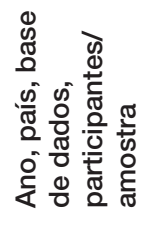 & 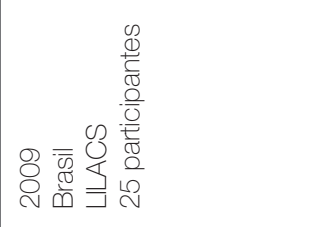 & 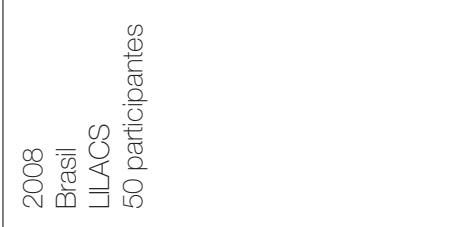 \\
\hline $\begin{array}{l}\frac{\stackrel{2}{0}}{\frac{0}{0}} \\
\frac{0}{0}\end{array}$ & 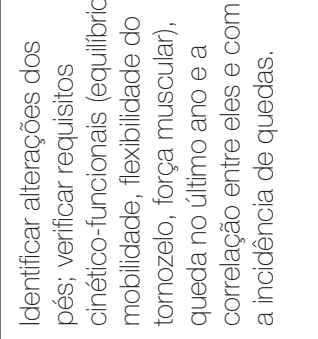 & 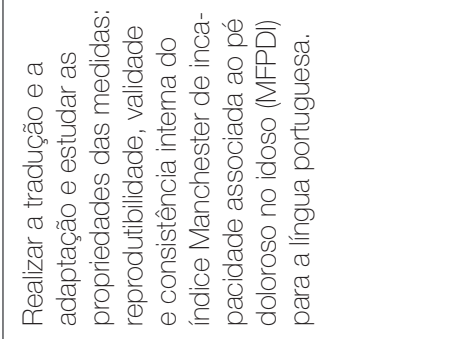 \\
\hline 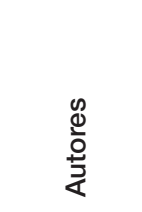 & 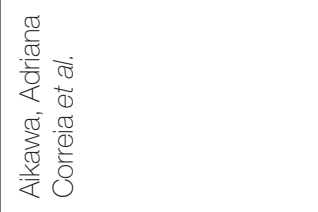 & 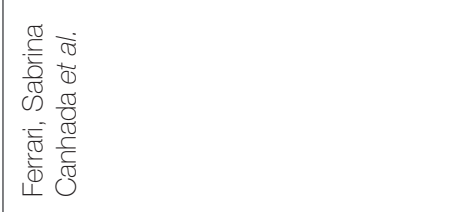 \\
\hline 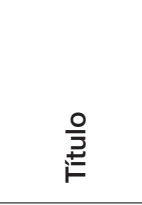 & 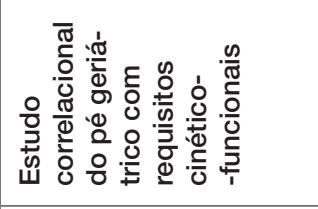 & 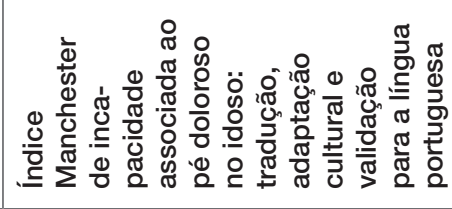 \\
\hline$\stackrel{\circ}{z}$ & $\stackrel{\infty}{\circ}$ & 8 \\
\hline
\end{tabular}




\begin{tabular}{|c|c|}
\hline 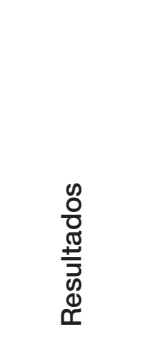 & 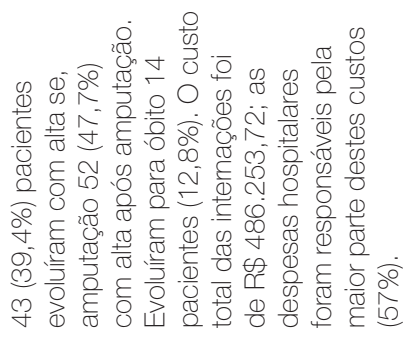 \\
\hline 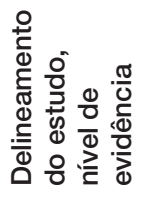 &  \\
\hline 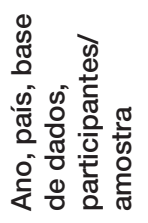 & 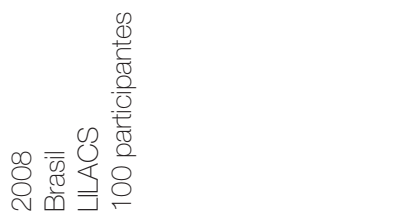 \\
\hline$\frac{\stackrel{0}{2}}{\stackrel{0}{0}}$ & 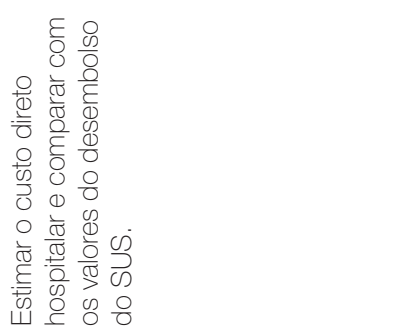 \\
\hline \begin{tabular}{l}
$\mathscr{8}$ \\
$\frac{1}{0}$ \\
\multirow{2}{*}{}
\end{tabular} & 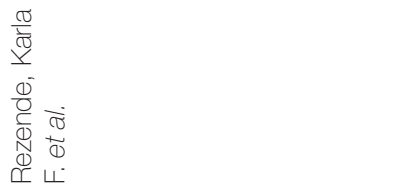 \\
\hline 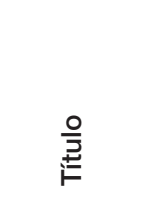 & 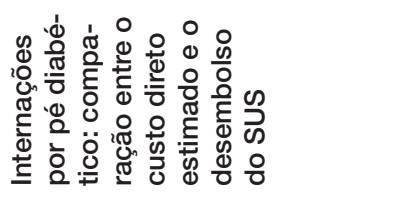 \\
\hline$\stackrel{\circ}{z}$ & 우 \\
\hline
\end{tabular}


O fato da publicação de artigos alusivos às alterações nos pés dos idosos ser mais recente pode estar relacionado ao aumento da população idosa nos últimos anos e nos remete ao ano de 2006, quando foi concluído o processo de revisão e atualização da Portaria n 1.395/GM, de 10 de dezembro de 1999 sendo aprovada a Política Nacional de Saúde da Pessoa Idosa (PNSPI), que tem por finalidade "recuperar, manter e promover a autonomia e a independência dos indivíduos idosos, direcionando medidas coletivas e individuais de saúde para esse fim, em consonância com os princípios e diretrizes do Sistema Único de Saúde (SUS)" (BRASIL, 2006).

Alguns estudos relacionam as alterações podais ao desenvolvimento de doenças reumáticas, complicações da insuficiência vascular, presença de doenças degenerativas que diminuem a amplitude do movimento e à obesidade (PINTO, 2012; FERRARI et al., 2009; YI et al., 2014).

Assim, para recuperar, manter e/ou promover a autonomia e independência dos idosos, os pés devem também ser foco de cuidados na atenção à saúde, pois desempenham importante função no controle da postura, manutenção da estabilidade e execução dos movimentos.

Para proceder à discussão, os artigos foram separados por categorias, como pode ser observado no Quadro 2. 
Quadro 2 - Separação dos artigos por categorias

\begin{tabular}{|c|c|}
\hline Categorias & Artigos \\
\hline \multirow{8}{*}{$\begin{array}{l}\text { 1. Alterações nos pés relacionadas à } \\
\text { incapacidade funcional no idoso }\end{array}$} & $\begin{array}{l}\text { Alterações nos pés do idoso hospitalizado: um olhar } \\
\text { cuidadoso da enfermagem }\end{array}$ \\
\hline & $\begin{array}{l}\text { Limitação funcional relacionada ao pé doloroso em } \\
\text { idosos }\end{array}$ \\
\hline & $\begin{array}{l}\text { Estudo correlacional do pé geriátrico com requisitos } \\
\text { cinético-funcionais }\end{array}$ \\
\hline & $\begin{array}{l}\text { Alterações podais e mobilidade em idosos atendidos } \\
\text { em um ambulatório de geriatria }\end{array}$ \\
\hline & $\begin{array}{l}\text { Caracterização dos problemas relacionados aos pés de } \\
\text { idosos de uma comunidade em município do interior do } \\
\text { Estado de São Paulo }\end{array}$ \\
\hline & $\begin{array}{l}\text { Pé doloroso do idoso associado à incapacidade } \\
\text { funcional }\end{array}$ \\
\hline & $\begin{array}{l}\text { Prevalencia de patología del pie en una población geriá- } \\
\text { trica y su impacto en la función, la marcha y el síndrome } \\
\text { de caídas }\end{array}$ \\
\hline & $\begin{array}{l}\text { Índice Manchester de incapacidade associada ao pé } \\
\text { doloroso no idoso: tradução, adaptação cultural e } \\
\text { validação para a língua portuguesa }\end{array}$ \\
\hline \multirow{2}{*}{$\begin{array}{l}\text { 2. Hospitalizações em decorrência } \\
\text { de alteração podal: o pé diabético na } \\
\text { população idosa }\end{array}$} & $\begin{array}{l}\text { Educação terapêutica para diabéticos: os cuidados } \\
\text { com os pés na realidade de pacientes e familiares }\end{array}$ \\
\hline & $\begin{array}{l}\text { Internações por pé diabético: comparação entre o } \\
\text { custo direto estimado e o desembolso do SUS }\end{array}$ \\
\hline
\end{tabular}

Fonte: Elaborado pelas autoras.

\subsection{Alterações podais relacionadas à incapacidade funcional no idoso}

Os estudos apontam que as alterações podais têm grande influência na capacidade funcional dos idosos. As alterações mais frequentes nos pés dos idosos que participaram das pesquisas foram: hálux valgo, deformidades nos pequenos artelhos, calosidades, ressecamento, fissuras, unhas quebradiças, encravadas e compridas (SILVA; ESPÍRITO SANTO; CHIBANTE, 2017; BARBOSA et al., 2015; MARIN; MACIEL, 2014; PRATO; SANTOS; TREVISANI, 2012; GALLARDO-PRIETO; GALINDO; DESES, 2012; AIKAWA et al., 2009; FERRARI et al., 2008). 
Um estudo brasileiro cujo objetivo foi verificar as alterações podais mais frequentes e o prejuízo destas na mobilidade de idosos em atendimento ambulatorial, envolveu 121 idosos, em que 85,1\% dos pacientes (103) eram do sexo feminino, e mostrou que as alterações mais frequentes foram espessamento ou calosidades em face plantar, encontradas em $60,3 \%$ dos idosos (n=3), hálux valgo com deformidade, que afetou $45,5 \%$ dos idosos $(n=55)$ e deformidades nos artelhos, encontradas em $43 \%$ dos idosos $(n=52)$ (BARBOSA et al., 2015). Resultados próximos obteve o estudo realizado na Espanha envolvendo 171 idosas: 65,5\% (n=112) apresentaram deformidades nos artelhos e 61,3\% $(n=105)$ apresentaram hálux valgo, hálux rígido e/ou hálux interfalângico (GALLARDO-PRIETO; GALINDO; DESES, 2012).

De modo geral, as mulheres desenvolvem e relatam mais problemas nos pés do que os homens. Isto pode ser atribuído à influência dos calçados de salto alto e bico fino, que aumentam a chance de desenvolvimento de problemas no pé com o avanço da idade. Outro aspecto é a tolerância à dor; as mulheres relatam mais as dores que os homens e, consequentemente, procuram mais os serviços de saúde (PRATO; SANTOS; TREVISANI, 2012).

O uso de calçados inadequados também é comum entre os idosos, assim como entre adultos jovens. A população mais jovem usa calçados inadequados como calçados com saltos e bico fino por modismo e estética, a despeito do conforto, da segurança e, em longo prazo, dos danos causados aos pés. Contrária à população mais jovem, os mais idosos usam calçados inadequados como sapatilhas de pano, rasteiras, muitas vezes não antiderrapante e preferem estes principalmente porque buscam conforto, ainda que em detrimento à segurança. Um estudo realizado em um ambulatório de Geriatria e Gerontologia entre 2007 e 2008 com uma amostra de 50 idosos, mostrou que apenas $29 \%$ dos indivíduos (14) faziam uso do calçado considerado adequado e seguro para os idosos. A maioria dos indivíduos fazia uso de calçados inadequados, sendo esses, principalmente, do tipo sapatilhas, sandália, chinelo e mule (BARBOSA et al., 2015).

Confirmando os achados do estudo anterior, uma pesquisa realizada em uma unidade de Saúde da Família no interior do Estado de São Paulo no ano de 2012, destacou que a intensidade e a complexidade dos problemas encontrados nos pés dos idosos foi alarmante quando se observou que, com frequência esses idosos utilizam calçados inadequados. Alterações como deformidades das unhas e proeminências ósseas, entre outras, impediam o uso de calçado adequado (MARIN; MACIEL, 2014).

Os idosos geralmente escolhem o tipo de calçado com material macio e estrutura flexível, que parece ser mais confortável, e acomodam melhor 
as deformidades dolorosas dos pés e tais fatos poderiam concorrer para as escolhas inadequadas de calçados, pois outros fatores são mais importantes, como segurança (PRATO; SANTOS; TREVISANI, 2012).

Destaca-se também nos estudos a prevalência do pé doloroso que interfere nas atividades de movimentação, trabalho e lazer dos idosos, além de provocar incômodo e preocupação (OLIVEIRA et al., 2015; BARBOSA et al., 2015; MARIN; MACIEL, 2014; PRATO; SANTOS; TREVISANI, 2012; FERRARI et al., 2008).

Em um estudo houve correlação significativa entre a intensidade da dor e a incapacidade funcional associada ao pé doloroso quando a dor considerada era em movimento. A frequência de incapacidade funcional associada ao pé doloroso no idoso utilizando o Manchester Foot Pain and Disability Index (MFPDI) foi > 50\% em uma amostra de 100 idosos (PRATO; SANTOS; TREVISANI, 2012). Em outro estudo, dos 121 idosos entrevistados 94,2\% ( $n=114)$ referiram dor (BARBOSA et al., 2015).

Os problemas nos pés dos idosos interferem especialmente na limitação de movimentos, essencial para a manutenção da qualidade de vida dessas pessoas. Acrescenta-se o fato de a maioria dos acometidos serem idosas e comumente apresentarem maior vulnerabilidade nas condições de saúde, principalmente quando pertencem às classes socioeconômicas pouco favorecidas, o que dificulta o acesso ao necessário atendimento à saúde especialmente por se tratar de uma condição pouco valorizada, como é o caso dos cuidados com os pés (MARIN; MACIEL, 2014), que envolvem a orientação quanto ao calçado correto, o corte correto das unhas, desbastamento de calos e calosidades e retirada de verruga plantar por profissional capacitado, entre outros cuidados.

As alterações encontradas nos pés de pessoas idosas são de grande relevância e interesse para a prática clínica, porque podem determinar consequências trágicas, que vão desde o comprometimento da mobilidade até o favorecimento de quedas que colocam em risco a independência dos idosos (FERRARI et al., 2008; SILVA; ESPÍRITO SANTO; CHIBANTE, 2017).

\subsection{Hospitalizações em decorrência de alteração podal - pé diabético na população idosa no Brasil}

Apesar dos muitos estudos abordando o pé diabético e apresentando evidências sobre asas ações preventivas no combate às suas complicações, ainda é precária a condição de assistência aos portadores de diabetes pois ainda é focada no atendimento médico, não envolvendo o trabalho inter ou multidisciplinar e nesta assistência, muitas vezes há falta de integração dos 
profissionais com os familiares dos portadores de diabetes. Estudos mostram que essa patologia é mais frequente na população idosa, e por isso esta ocorrência mereceu destaque nesta revisão (SALCI; MEIRELLES; SILVA, 2017; SILVA; ESPÍRITO SANTO; CHIBANTE, 2017; CISNEROS; GONÇALVES, 2011; REZENDE et al., 2008).

Alguns fatores contribuem para o risco de complicações do diabetes em idosos. A diminuição da acuidade visual, seja pela doença ou pelo próprio processo de envelhecimento, impede a visualização de degraus e obstáculos além de dificultar a realização do autoexame e do autocuidado com os pés. Além disso, a diminuição da acuidade visual está associada ao impacto negativo sobre a qualidade de vida, refletindo-se na autoestima, status social e atividades ocupacionais, podendo desencadear problemas psicológicos, sociais e econômicos (MARIN; MACIEL, 2014).

Idosos que vivem sozinhos ou que se isolam socialmente se tornam mais suscetíveis a desenvolver complicações por não contarem com apoio, no caso de necessidade, de alguém que lhes dê suporte nos seus cuidados diários relacionados à alimentação, uso de medicamentos e aos cuidados mais especializados com os pés.

O pé diabético é hoje uma preocupação de ordem mundial, que envolve alto custo humano e financeiro, caracterizado como um conjunto de alterações neurológicas, ortopédicas, vasculares e/ou infecciosas, ocorridas isoladamente ou em conjunto nos pés de pacientes diabéticos (CAIAFA et al., 2011).

A visão corrente hoje de que a presença de pelo menos uma dessas alterações nos pés de pacientes diabéticos é suficiente para classificar este membro como pé diabético é definitivamente contrária à visão anterior de um pé que já apresente lesões geralmente provocadas por traumas. Um pé ulcerado, necrosado e infectado é consequente da ausência de uma abordagem adequada com orientações para o autocuidado do paciente diabético e da falta de capacitação de profissionais no atendimento a este paciente (BONA et al., 2010).

Diretrizes da Sociedade Brasileira de Diabetes (SBD, 2019) estimam que em 2017 a população mundial de diabéticos era de aproximadamente 424,9 milhões de pessoas e deverá atingir 628,6 milhões em 2045.

Os estudos que constam nesta revisão integrativa selecionados para esta categoria abordam especificamente o pé diabético, suas complicações, o conhecimento dos pacientes diabéticos sobre essas complicações e sobre os cuidados com os pés e o custo direto relacionado às internações por pé diabético. Nestes dois estudos houve predominância de idosos entre a sexta e oitava décadas de vida (56,6\% e 78\%) (CISNEROS; GONÇALVES, 2011; REZENDE et al., 2008), corroborando as diretrizes da SBD (2019), que associa o aumento da prevalência 
de diabetes a diversos fatores, como rápida urbanização, transição epidemiológica, transição nutricional, maior frequência de estilo de vida sedentário, maior frequência de excesso de peso, à maior sobrevida dos indivíduos com diabetes e, também ao crescimento e envelhecimento populacional.

Um estudo quantitativo realizado em um hospital de emergência em São Luíz (MA) visando identificar características, conhecimento e autocuidado de idosos portadores de DM com úlceras nos pés, envolveu 20 idosos e obteve resultados semelhantes: a faixa etária predominante foi entre 71 e 80 anos (40\%), seguida de 60 a 65 anos (35\%) sendo metade do sexo feminino e metade do sexo masculino. Quanto a escolaridade, 55\% não sabiam ler e escrever, 50\% percebiam renda familiar de até 1 salário mínimo. Quanto ao autocuidado, 12 idosos enxugavam e hidratavam os pés, 13 cortavam as unhas com tesouras e 4 cortavam com gilete e faca (PEREIRA et al., 2010). Os resultados mostraram que as lesões nos membros inferiores são mais frequentes em pacientes idosos diabéticos com baixa escolaridade e baixa renda. Isto pode indicar dificuldades na adoção de medidas necessárias para o controle da doença e prevenção das complicações, como por exemplo, manter a dieta recomendada, dificuldades no entendimento das orientações quanto ao tratamento medicamentoso, entre outros (PEREIRA et al., 2010).

O desenvolvimento do DM e, consequentemente, de suas complicações, não está associado à escolaridade e pode acometer pessoas de todos os níveis socioeconômicos. Contudo, a baixa escolaridade pode favorecer a não adesão ao plano terapêutico pela dificuldade de ler e entender a prescrição, o que aumenta os riscos à saúde (OLIVEIRA; ZANETTI, 2011; RODRIGUES et al., 2012).

As alterações mais frequentes nos pés dos idosos foram: pele ressecada, unhas grossas, rachaduras e ranhuras. Assim, queixas relativas não afastam a necessidade de um exame minucioso dos pés, pois geralmente os pacientes não valorizam problemas importantes como calos, rachaduras, micoses, entre outros (PEREIRA et al., 2010; CAIAFA et al., 2011).

No estudo desenvolvido em São Luíz (MA), 75\% dos idosos faziam acompanhamento na rede pública de saúde e $60 \%$ tinham pouca informação sobre a doença. Isto ratifica o discurso dos autores do estudo realizado no Estado de Sergipe que afirmaram que poucos pacientes recebem orientação para o autocuidado com os pés e/ou têm seus pés examinados nas consultas, elevando as taxas de complicações, internações, amputações e mortes relacionadas o pé diabético em vários lugares do país (PEREIRA et al., 2010; REZENDE et al., 2008).

Tendo em vista que o melhor tratamento para o pé diabético é sua prevenção, as ações educativas para o autocuidado são indispensáveis. A adesão às ações preventivas é pequena em virtude da evolução silenciosa das complicações 
do diabetes. Assim, para muitos pacientes a importância da prevenção só é evidenciada após experiência própria ou alheia de uma complicação. Portanto o emprego de Equipes Multidisciplinares é essencial para o cuidado integral a esses pacientes e seus familiares (CISNEROS; GONÇALVES, 2011; CAIAFA et al., 2011).

Relatos como liberdade cerceada por terceiros, dificuldade física, necessidade de ajuda e situação de risco nos autocuidados chamam a atenção em um estudo que envolveu 30 pacientes. Para $19(63,4 \%)$ indivíduos a participação da família é positiva no controle do diabetes e ajuda nos cuidados com os pés prevenindo complicações, já que muitos idosos têm dificuldade física e/ou visual, o que aumenta o receio de provocar algum ferimento nos pés durante o corte das unhas, por exemplo. No entanto, 11 (36,6\%) indivíduos perceberam essa participação como forma de controle da família sobre sua autonomia e independência, provocando o conflito familiar (CISNEROS; GONÇALVES, 2011).

Ressalta-se a valorização de uma abordagem mais humanizada, com equipes interdisciplinares que atuem no cuidado integral e desenvolvam ações de educação em saúde tanto para os pacientes, quanto para os familiares, com abordagem biopsicossocial, acolhendo, esclarecendo as dúvidas, indo de encontro às demandas de saúde, orientando e auxiliando na resolução dos conflitos.

Em relação ao desfecho das internações por complicações com o pé diabético no Estado de Sergipe, 14 (12,8\%) dos 109 pacientes evoluíram para óbito e $95(87,2 \%)$ receberam alta; 93 (85\%) pacientes foram submetidos ao tratamento cirúrgico: 58 (62,4\%) sofreram amputações e 35 (37,6\%) passaram por desbridamentos. Realizaram procedimento de revascularização fêmoro-poplítea cinco (4,6\%) pacientes (REZENDE et al., 2008).

Assim, ações como exame periódico dos pés para avaliação dos riscos e capacitação de profissionais de saúde para os cuidados específicos com os pés de pacientes diabéticos, e mais ainda para idosos diabéticos, que constituem a maior parte das internações por pé diabético no SUS, devem ser potencializadas a fim de diminuir o número de internações e amputações, reduzindo os gastos com essas complicações.

\section{Conclusão}

Os resultados desta revisão apontaram que as alterações nos pés dos idosos são frequentes e podem ocorrer tanto como resultado de maus tratos aos pés, como uso de calçados inadequados e traumas, como em consequência de doenças como DM. As alterações podais mais frequentes foram hálux valgo, 
deformidades nos pequenos artelhos, calosidades, ressecamento, fissuras, unhas quebradiças, encravadas e compridas.

As lesões nos membros inferiores são mais frequentes em pacientes idosos diabéticos com baixa escolaridade e baixa renda. Foi possível observar que, dos idosos portadores de pé diabético que faziam acompanhamento na rede pública de saúde a maior parte tinha pouca informação sobre o DM e suas complicações. Além disso, percebeu-se que poucos profissionais dão a devida importância ao pé diabético, visto que em uma das pesquisas poucos pacientes relataram ter seus pés examinados durante as consultas e grande parte das internações por pé diabético ocorreu em pacientes entre a sexta e a oitava década de vida, que apresentam baixa escolaridade e baixa renda.

A adesão às ações preventivas é pequena em virtude da evolução silenciosa das complicações do diabetes. Assim, para muitos pacientes a importância da prevenção só é evidenciada após experiência própria ou alheia de uma complicação.

Portanto, são necessárias ações voltadas para conscientização dos pacientes e capacitação dos profissionais de saúde visando à manutenção da saúde dos pés, prevenção e/ou tratamento das alterações, além da reabilitação dos pacientes. A caracterização do paciente é imprescindível para desenvolver estratégias para conscientização, prevenção e tratamento, com o intuito de alcançar o entendimento e as condições financeiras desses pacientes, para que ele seja capaz de realizar o autocuidado.

O enfermeiro com treinamento específico para cuidar dos pés de idosos com alterações é o profissional mais indicado para prestar o cuidado a esses pacientes, orientando para o autocuidado, intervindo no desenvolvimento do processo de incapacidade e dependência no idoso intensificado por essas alterações.

Neste contexto, a consulta de enfermagem apresenta-se como um fator importante de proteção ao agravo das alterações podais, visto que aproxima profissional e paciente contribuindo para a forma de cuidar e educar.

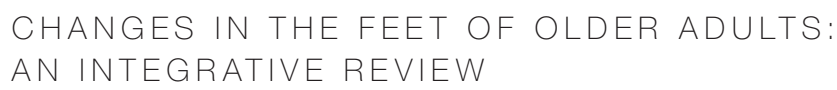
the older population and considering how changes in the feet can interfere with activities of daily living, and consequently, the quality of 
life of these older adults, the present study aimed to: identify in the literature scientific evidence about most frequent changes in the feet of older adults. The following research question was raised: How has the literature approached the subject of foot changes in older adults? This is an Integrative Review, and for this purpose a search was made for articles published and indexed in the Virtual Health Library using the combination of the following Health Sciences Descriptors in Portuguese: "Foot" and "Elderly" accompanied by the Boolean operator "and". The final result of this survey consists of ten articles published between 2008 and 2017 in two databases: Latin American and Caribbean Health Sciences Literature (LILACS) and Online Medical Literature Analysis and Retrieval System (MEDLINE). The articles were divided into two categories: Changes in the feet related to disability in older adults; Hospitalizations due to foot alteration: the diabetic foot in the older population. Changes in the feet of older adults are frequent and may occur as a result of foot abuse, such as wearing inappropriate shoes and trauma, and as a consequence of diseases such as diabetes mellitus. The most common foot changes were hallux valgus, small toe deformities, callus, dryness, fissures, brittle, ingrown and long nails. These changes may compromise mobility and even favor falls, jeopardizing the independence of older adults.

keywords

Feet. Older Adults. Geriatric Nursing.

referências

AlKAWA, Adriana Correia et al. Estudo correlacional do pé geriátrico com requisitos cinético-funcionais. Fisioterapia e Movimento, Curitiba, v. 22, n. 3, p. 395-405, jul./ set. 2009.

BARBOSA, Keylla Talitha Fernandes et al. Alterações podais e mobilidade em idosos atendidos em um ambulatório de geriatria. Revista de Pesquisa Cuidado é Fundamental Online, Rio de Janeiro, v. 7, n. 2, p. 2254-2262, abr./jun. 2015.

BONA, Socorro Ferreira et al. Prevalência do pé diabético nos pacientes atendidos na emergência de um hospital público terciário de Fortaleza. Revista Brasileira de Clínica Médica, São Paulo, v. 8, n. 1, p. 1-5, jan. 2010.

BRASIL. Ministério da Saúde. Portaria n. 2.528, de 19 de outubro de 2006. Aprova a Política Nacional de Saúde da Pessoa Idosa. Brasília, DF: Ministério da Saúde, 2006. Disponível em: https://bvsms.saude.gov.br/bvs/saudelegis/gm/2006/prt2528_19_10_2006. html. Acesso em: dez. 2019.

CAIAFA, Jackson Silveira et al. Atenção integral ao portador de pé diabético. Jornal Vascular Brasileiro, Porto Alegre, v. 10, n. 4, supl. 2, p. 1-32, 2011. 

para diabéticos: os cuidados com os pés na realidade de pacientes e familiares. Ciência \& Saúde Coletiva, Rio de Janeiro, v. 16, n. 1, p. 1505-1514, 2011

ERVATTI, Leila Regina; BORGES, Gabriel Mendes; JARDIM, Antonio de Ponte (org.). Mudança demográfica no Brasil no início do século XXI: subsídios para as projeções da população. Rio de Janeiro: IBGE, 2015. (Série Estudos \& Análises: Informação Demográfica e Socioeconômica, 3).

FERRARI, Sabrina Canhada et al. Índice Manchester de incapacidade associada ao pé doloroso no idoso: tradução, adaptação cultural e validação para a língua portuguesa. Revista Brasileira de Reumatologia, São Paulo, v. 48, n. 6, p. 335-341, dez. 2008.

FERRARI, Sabrina Canhada et al. Patologias no pé do idoso. Revista Brasileira de Ciências do Envelhecimento Humano, Passo Fundo, v. 6, n. 1, p. 106-118, jan./abr. 2009.

GALLARDO-PRIETO, Lorenza Martínez; GALINDO, Luis Felipe Hermida; DESES, Carlos d'Hyver de las. Prevalencia de patología del pie en una población geriátrica y su impacto en la función, la marcha y el síndrome de caídas. Revista Española de Geriatría y Gerontología, España, v. 47, n. 1, p. 19-22, enero/feb. 2012.

MARIN, Maria José Sanches; MACIEL, Mateus de Carvalho. Caracterização dos problemas relacionados aos pés de idosos de uma comunidade em município do interior do estado de São Paulo. Revista Brasileira de Geriatria e Gerontologia, Rio de Janeiro, v. 17, n. 2, p. 243-253, abr./jun. 2014.

MENDES, Karina Dal Sasso; SILVEIRA, Renata Cristina de Campos Pereira; GALVÃO, Cristina Maria. Revisão integrativa: método de pesquisa para a incorporação de evidências na saúde e na enfermagem. Texto \& Contexto - Enfermagem, Florianópolis, v. 17, n. 4, p. 758-764, out./dez. 2008.

OLIVEIRA, Fabiana Maria Rodrigues Lopes de et al. Limitação funcional relacionada ao pé doloroso em idosos. Revista da Rede de Enfermagem do Nordeste, Fortaleza, v. 16, n. 4, p. 586-592, 2015. Disponível em: http://periodicos.ufc.br/rene/article/ view/2751/2134. Acesso em: dez. 2019.

OLIVEIRA, Kelli Cristina Silva de; ZANETTI, Maria Lúcia. Conhecimento e atitude de usuários com diabetes mellitus em um Serviço de Atenção Básica à Saúde. Revista da Escola de Enfermagem da USP, São Paulo, v. 45, n. 4, p. 862-868, ago. 2011.

PEREIRA, Aldeides dos Santos et al. Características, conhecimento e autocuidado de idosos portadores de Diabetes Melittus com úlcera de pé. Revista de Pesquisa em Saúde, São Luís, v. 11, n. 2, p. 20-25, maio/ago. 2010.

PINTO, Maurílio José. Os pés do idoso e suas repercussões na qualidade de vida. In: FREITAS, Elizabete Viana de; PY, Ligia (ed.). Tratado de Geriatria e Gerontologia. 3. ed. Rio de Janeiro: Guanabara Koogan, 2012. p. 1169-1178.

PRATO, Sabrina Canhada Ferrari; SANTOS, Fânia Cristina; TREVISANI, Virgínia Fernandes Moça. Pé doloroso do idoso associado à incapacidade funcional. Revista Dor, São Paulo, v. 13, n. 1, p. 18-24, mar. 2012

REZENDE, Karla F. et al. Internações por pé diabético: comparação entre o custo direto estimado e o desembolso do SUS. Arquivos Brasileiros de Endocrinologia \& Metabologia, São Paulo, v. 52, n. 3, p. 523-530, abr. 2008.

RODRIGUES, Flávia Fernanda Luchetti et al. Relação entre conhecimento, atitude, escolaridade e tempo de doença em indivíduos com diabetes mellitus. Acta Paulista de Enfermagem, São Paulo, v. 25, n. 2, p. 284-290, 2012.

SALCI, Maria Aparecida; MEIRELLES, Betina Hörner Schlindwein; SILVA, Denise Maria Guerreiro Vieira da. Atenção às pessoas com diabetes mellitus na perspectiva do modelo de atenção às condições crônicas. Revista Latino-Americana de Enfermagem, São Paulo, v. 25, e2882, 2017. 
SILVA, Joziane Santos da; ESPÍRITO SANTO, Fátima Helena do; CHIBANTE, Carla Lube de Pinho. Alterações nos pés do idoso hospitalizado: um olhar cuidadoso da enfermagem. Escola Anna Nery, Rio de Janeiro, v. 21, n. 1, e20170010, 2017. Disponível em: https://www.scielo.br/scielo.php?pid=S1414-81452017000100210\&script=sci_ abstract\&tIng=pt. Acesso em: dez. 2019.

SOCIEDADE BRASILEIRA DE DIABETES (SBD). Diretrizes da Sociedade Brasileira de Diabetes: 2019-2020. São Paulo: Clannad Editora Científica, 2019. Disponível em: https://www.diabetes.org.br/profissionais/images/DIRETRIZES-COMPLETA-2019-2020. pdf. Acesso em: jan. 2019.

VERAS, Renato; OLIVEIRA, Martha. Linha de cuidado para o idoso: detalhando o modelo. Revista Brasileira de Geriatria e Gerontologia, Rio de Janeiro, v. 19, n. 6, p. 887-905, 2016.

YI, Liu Chiao et al. Influência do índice de massa corporal no equilíbrio e na configuração plantar em obesos adultos. Revista Brasileira de Medicina do Esporte, São Paulo, v. 20, n. 1, p. 70-73, fev. 2014.

Data de Submissão: 18/07/2016

Data de Aprovação: 21/04/2020 